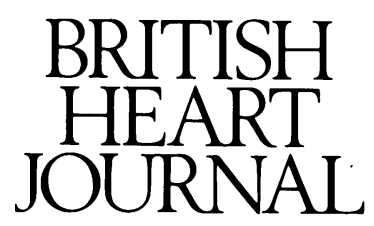

Editorial

\title{
Speeding up the review process
}

Over the past ten years the British Heart Journal has been a financial and scientific success. Changes in scientific publishing dictate that the Journal must change to maintain this position. A computerised tracking system for manuscripts and reviewers has now given us the opportunity to streamline the decision making process. This change will be welcomed by our contributors, some of whom perceive that the time taken between submission and final acceptance is too long.

The interval between submission and decision on an article has many components. A major factor is the time taken by reviewers to return reports. We all endorse peer review as the foundation of scientific publishing. Yet there is a price to pay, that of delay. The number of scientific articles written world-wide continues to rise, as does the use of reviewers' time. A review takes time and effort: a detailed review of a difficult article takes us at least an hour. Published data on reviewers activities suggest that many take longer. ${ }^{12}$ Why do reviewers review? The most frequently used argument is that reviewers being members of the scientific community have an academic duty to do this work. To the busy clinician this is not always a compelling argument. Some journals seek permission from the reviewer before sending an article: we do not have the office capacity to do this. Some reputable journals pay reviewers a modest sum, but this would be an unacceptable financial drain on the British Heart Journal.

In consultation with the Editorial Committee of the British Heart Journal we have decided on changes that will speed up the review process. Six additional United Kingdom members are being added to the committee, and representatives from mainland Europe and the United States of America are being proposed. The number of reviewers is to be increased. We are keen to capitalise on the specialist knowledge of lecturers, senior research fellows, and senior registrars within their own areas of interest.

The way in which submitted manuscripts are handled is to change. As before, the corresponding author will receive a postcard giving the date on which the article was received and a code number. But in future the paper will be sent to external reviewers and to at least one member of the editorial committee. Authors may suggest three suitable external reviewers. While we will normally use one or more of the suggested names we reserve the right not to do so.
The editorial committee members will comment rapidly on how appropriate the article is for the British Heart Journal and indicate the degree of importance while noting any obvious flaws. External reviewers will continue to make more detailed criticisms. The reports will be considered jointly by the editor and associate editors, and, where appropriate, one of the assistant editors and a statistical expert. We will meet regularly and will decide on the status of each article within twelve weeks of the submission date. We will be able to tell most authors the decision well within this time. If they wish, authors with fax machines can receive the answer even more quickly. The decision will be-accept, reject, or accept subject to specific revision by the authors within one month.

Articles will be revised less than in the past. In a sense the British Heart Journal by accepting an article gives it a stamp of approval, but if authors are pressed by the Journal to answer all the reviewers' criticisms they may end up writing an article that does not say what they originally intended. Fewer revisions may lead to a vigorous correspondence column pointing out different interpretations of a paper, and this is what we would like to encourage.

Any decision process should have an appeal mechanism. The selected reviewers may have been ill-informed on a particular topic. Our rejection letter will indicate whether the decision was based solely on reviewers' criticisms, or on broader issues of interest or suitability. Authors can resubmit, pointing out why they consider the review is unfair. A new set of reviewers will be used to assess such submissions.

The new system of handling submissions will depend heavily on the efforts of the office staff, the editors, and members of the Editorial Committee to maintain its momentum.

British Heart Journal,

9 Fitzroy Square,

London WIP 5 AH

M J DAVIES

Editor

KIM FOX

PETER MILLS

Associate editors

1 Lock S, Smith J. What do peer reviewers do? JAMA 1990;263:1341-3.

Yankauer A. Who are the peer reviewers and how much do they review? $J A M A$ 1990;263:1338-40. 